\title{
Inverse problem for a nonlinear Helmholtz equation
}

\section{Problème inverse pour une équation de Helmholtz non linéaire}

\author{
Emmanuel Jalade \\ Université de Provence C.M.I., U.M.R. 6632, 39, rue Joliot-Curie, 13453 Marseille cedex 13, France
}

Received 10 October 2002; accepted 2 July 2003

Available online 16 December 2003

\begin{abstract}
This paper is devoted to the uniqueness of the coefficients $\theta, \varphi \in L^{\infty}\left(\mathbb{R}^{3}\right)$, and $\psi \in L^{\infty}\left(\mathbb{R}^{3}, \mathbb{R}^{3}\right)$ for the nonlinear Helmholtz equations $-\Delta v(x)-k^{2} v(x)=\theta(x) v(x) F(|v(x)|)$ and $-\Delta v(x)-k^{2} v(x)=(\varphi(x) v(x)+i \psi(x) . \nabla v(x))|\nabla v(x)|^{r}|v(x)|^{s}$. For small values of $\lambda$, a solution $v$ is uniquely constructed by adding a small outgoing perturbation to the plane wave $x \rightarrow \lambda e^{i k x}$.d, where $|d|=1$ and $\lambda \geqslant 0$. We can write $v=v(x, \lambda, d)=\lambda e^{i k x . d}+u_{\infty}^{s}(x /|x|, d, \lambda) e^{i k|x|} /|x|+\mathrm{O}\left(1 /|x|^{2}\right)$ for large $|x|$. For a fixed $k>0$, we would like to prove that $\theta, \varphi$ and $\operatorname{div} \psi$ can be uniquely reconstructed from the behaviour of $u_{\infty}^{s}(x /|x|, d, \lambda)$ as $\lambda \rightarrow 0$. We prove the uniqueness in this paper.

(c) 2003 Elsevier SAS. All rights reserved.
\end{abstract}

\section{Résumé}

L'objet de ce papier est d'étudier l'unicité des coefficients $\theta, \varphi \in L^{\infty}\left(\mathbb{R}^{3}\right)$, et $\psi \in L^{\infty}\left(\mathbb{R}^{3}, \mathbb{R}^{3}\right)$ pour les Équations de Helmholtz non linéaires $-\Delta v(x)-k^{2} v(x)=\theta(x) v(x) F(|v(x)|)$ et $-\Delta v(x)-k^{2} v(x)=(\varphi(x) v(x)+i \psi(x) . \nabla v(x))|\nabla v(x)|^{r}|v(x)|^{s}$. Quand $\lambda$ est assez petit, on construit de manière unique une solution $v$ en ajoutant à l'onde incidente plane $x \rightarrow \lambda e^{i k x . d}(|d|=1$, $\lambda \geqslant 0)$ une perturbation $k$-sortante. On peut ainsi écrire $v=v(x, \lambda, d)=\lambda e^{i k x . d}+u_{\infty}^{s}(x /|x|, d, \lambda) e^{i k|x|} /|x|+\mathrm{O}\left(1 /|x|^{2}\right) \mathrm{quand}$ $|x| \rightarrow+\infty$. Considérons que $k>0$ est fixé. Le problème abordé dans ce papier est de montrer que $\theta, \varphi$ et div $\psi$ peuvent être construits de manière unique à partir du comportement asymptotique de $u_{\infty}^{s}(x /|x|, d, \lambda)$ quand $\lambda \rightarrow 0$. Nous démontrons l'unicité pour ce problème inverse, et développerons quelques aspects concernant la reconstruction.

(c) 2003 Elsevier SAS. All rights reserved.

MSC: 35R30; $35 \mathrm{~J} 60$

Keywords: Nonlinear Helmholtz equation; Inverse problem

E-mail address: jalade@cmi.univ-mrs.fr (E. Jalade). 


\section{Introduction}

Let $(\theta, \varphi, \psi) \in L^{\infty}\left(\mathbb{R}^{3}\right) \times L^{\infty}\left(\mathbb{R}^{3}\right) \times L^{\infty}\left(\mathbb{R}^{3}, \mathbb{R}^{3}\right)$, and assume the supports of these functions, supp $\theta$, supp $\varphi$ and supp $\psi$ are included in a fixed Euclidean ball $U=B\left(0, R_{0}\right) \subset \mathbb{R}^{3}$. We consider an odd function $F: \mathbb{C} \rightarrow \mathbb{C}$ such that $F(z)=z|z|^{p}(1+\varepsilon(|z|))$, where $p>0$ and $\varepsilon$ is a complex valued function that satisfies $\varepsilon \in C^{1}([0,+\infty[)$ (in fact, it suffices to assume that $\varepsilon \in C^{1}(] 0,+\infty[), \lim _{t \rightarrow 0} \varepsilon(t)=0$ and $\left.\lim _{t \rightarrow 0} t^{p+1} \varepsilon^{\prime}(t)=0\right)$.

We consider the nonlinear Helmholtz equations in $\mathbb{R}^{3}$

$$
-\Delta v-k^{2} v=\theta F(v)
$$

and

$$
-\Delta v-k^{2} v=(\varphi v+i \psi \cdot \nabla v)|\nabla v|^{r}|v|^{s},
$$

where $r \in\{0\} \cup[1,2], s \in\{0\} \cup[1,+\infty[$ and $r+s>0$.

Theses two equations are satisfied by the spatial part $v$ of time-harmonic solutions $w(x, t)=v(x) e^{i k t}$, with circular frequency $k>0$, of the respective equations

$$
\begin{aligned}
& \frac{\partial^{2} w}{\partial t^{2}}(x, t)-\Delta w(x, t)=\theta(x) F(w(x, t)), \\
& \frac{\partial^{2} w}{\partial t^{2}}(x, t)-\Delta w(x, t)=(\varphi(x) w(x, t)+i \psi(x) . \nabla w(x, t))|\nabla w(x, t)|^{r}|w(x, t)|^{s},
\end{aligned}
$$

with $(x, t) \in \mathbb{R}^{3} \times \mathbb{R}$.

Eqs. (1) and (2) can be viewed as linear homogeneous Helmholtz equation on $\mathbb{R}^{3}$, perturbed with a localized nonlinear term. Hence, by analogy with exterior problems and the Helmholtz equation perturbed by a short-range potential, we search solutions $v$ in the form

$$
v(x, d, \lambda)=\lambda e^{i k x . d}+u(x, d, \lambda), \quad \lambda \geqslant 0, d \in S^{2},
$$

where $u$ satisfies the so-called Sommerfeld $k$-outgoing condition. Consequently, $u$ will have the following asymptotic development

$$
u(x, d, \lambda)=\frac{e^{i k|x|}}{|x|}\left(u_{\infty}\left(\frac{x}{|x|}, d, \lambda\right)+\mathrm{O}\left(\frac{1}{|x|}\right)\right) \quad \text { as }|x| \mapsto \infty .
$$

We call $u_{\infty}$ the scattering amplitude of $u$, by analogy with the linear Helmholtz equation. For the linear case, one only considers $\lambda=1$; in this case, the scattering amplitude is often denoted $u_{\infty}(x /|x|, d, k)$, where the dependency in $k$ appears. This notation should not be confused with that introduced below.

Let us briefly describe the organization of this paper.

In Section 1, we study the two following equations (cf. Proposition 2)

$$
-\Delta u-k^{2} u=\theta F\left(u+\lambda e^{i k x \cdot d}\right)
$$

and

$$
-\Delta w-k^{2} w=\left(\varphi\left(w+\lambda e^{i k x \cdot d}\right)+i \psi \cdot \nabla\left(w+\lambda e^{i k x \cdot d}\right)\right)\left|\nabla\left(w+\lambda e^{i k x \cdot d}\right)\right|^{r}\left|w+\lambda e^{i k x \cdot d}\right|^{s} .
$$

Section 2 is devoted to the behaviour of $u$ and $w$ as $\lambda \rightarrow 0$.

In Section 3, we show $\theta$ (respectively $\varphi$ and $\operatorname{div} \psi$ ) is uniquely determined from the knowledge of $\lim _{\lambda \rightarrow 0} u_{\infty}(\hat{x}, d, \lambda) / \lambda^{p+1}$ (respectively $\lim _{\lambda \rightarrow 0} w_{\infty}(\hat{x}, d, \lambda) / \lambda^{r+s+1}$ ) (see Lemma 3 ), for $(\hat{x}, d)$ lying in a subset of $S^{2} \times S^{2}$ (Theorems 1 and 2). If $\theta$ (respectively $\varphi, \psi$ ) is assumed to be a radial function, we can improve the latter result. Note that we only consider a fixed $k>0$, except in Section 3.2.3 in which we consider $k \rightarrow \infty$ for an approximated reconstruction. We finally provide a generalisation of these last results for the equation $-\Delta u(x)-k^{2} u(x)=u(x) \sum_{n=1}^{+\infty} a_{n}(x)|u(x)|^{n}$, with $\sum_{n=1}^{+\infty}\left\|a_{n}\right\|_{L^{\infty}} t^{n}<+\infty$, for some $t>0$. 
This inverse problem is analogous with those concerning the linear Helmholtz equation with a short-range potential (see [5]) and the wave equation outside a smooth obstacle (see [1]); the potential and the obstacle can be uniquely recovered from the scattering amplitude, for all incident directions and a countable set of incident directions respectively (see the above references).

A similar inverse problem associated with the following nonlinear Schrödinger equation

$$
i \frac{\partial u}{\partial t}-\Delta u=\theta u|u|^{p},
$$

has been investigated by several authors via the Scattering Operator that can be defined for a small initial datum (see [6] and [7]). Eq. (1) is satisfied by the spatial part of the time-harmonic solutions of Eq. (8), with circular frequency $k^{2}>0$. An explicit reconstruction of $\theta$ is given in [6] (and [7] for a more general situation) from the nonlinear Scattering Operator. Here we choose to use instead the scattering amplitude $u_{\infty}$ defined in (5).

In fact, contrary to the Scattering Operator associated with the linear Schrödinger equation, the nonlinear Scattering Operator does not seem to be directly connected to the scattering amplitude $u_{\infty}$ defined in (5).

Many authors have also considered inverse problems associated with nonlinear elliptic equations different from the nonlinear Helmholtz equation (see [3,4]). In [4] (and [3] for the two-dimensional case), the authors show the uniqueness for the reconstruction of the nonlinear part of the operator $-\Delta+a(., u)$ acting on a smooth bounded domain from the knowledge of the Dirichlet-to-Neumann map.

\section{Notations}

For $x, y \in \mathbb{R}^{3}, x . y$ will denote the usual scalar product that defines the Euclidean norm $|x|=\sqrt{x . x}$ on $\mathbb{R}^{3}$. This notation will be extended to complex vectors $x, y \in \mathbb{C}^{3}$, but is not be confused with the Hermitian product on $\mathbb{C}^{3}$.

For $m \in \mathbb{R}^{3}$ and $0<r<r^{\prime}, B(m, r)$ is the Euclidean ball centered at $m$ and of radius $r$; its boundary is denoted $S(m, r)=\partial B(m, r)$ and $C\left(m, r, r^{\prime}\right)=\left\{x \in \mathbb{R}^{3}, r<|x-m|<r^{\prime}\right\}$. For the sake of simplicity, we set $S^{2}=S(0,1)$. More generally, if $(\mathcal{H},\|\|)$ is a Hilbert space and for $u \in \mathcal{H}, r>0$, we denote $B_{\mathcal{H}}(u, r)$ the Hilbertian ball centered at $u$ and of radius $r$.

For any subset $W \subset \mathbb{R}^{3}, \chi_{W}$ denotes the characteristic function of $W$.

We define $C_{c}^{\infty}\left(\mathbb{R}^{3}\right)$ as the set of smooth test functions on $\mathbb{R}^{3}$ with a bounded support. For $1 \leqslant p \leqslant+\infty$ and $n \in \mathbb{Z}, L^{p}=L^{p}\left(\mathbb{R}^{3}\right)$ and $W^{n, p}=W^{n, p}\left(\mathbb{R}^{3}\right)$ denote the usual Sobolev spaces. We define $W_{\text {loc }}^{n, p}=W_{\text {loc }}^{n, p}\left(\mathbb{R}^{3}\right)$ to be the set of measurable functions $u$ such that $\varphi u \in W^{n, p}$ for all $\varphi \in C_{c}^{\infty}\left(\mathbb{R}^{3}\right)$. We denote $H^{n}=W^{n, 2}$ and $H_{\mathrm{loc}}^{n}=W_{\mathrm{loc}}^{2, p}$. For $u \in C_{c}^{\infty}\left(\mathbb{R}^{3}\right), \mathcal{F}(u)$ is the Fourier transform of $u$ defined by

$$
\mathcal{F}(u)(\xi)=\int_{\mathbb{R}^{3}} e^{-i x \cdot \xi} u(x) d x .
$$

For any distribution $u=\left(u_{1}, u_{2}, u_{3}\right)$, we define the divergence operator, that is $\operatorname{div} u=\sum_{j=1}^{3} \frac{\partial u_{j}}{\partial x_{j}}$.

We will use the space $B$ and its associated dual $B^{*}$ that are defined in [2] (Chapter 14.1) and satisfy $C_{c}^{\infty}\left(\mathbb{R}^{3}\right) \subset B \subset L^{2}\left(\mathbb{R}^{3}\right) \subset B^{*} \subset L_{\text {loc }}^{2}\left(\mathbb{R}^{3}\right)$ with bounded imbeddings. We recall the norms

$$
\|u\|_{B}=\sum_{j \geqslant 1}\left(2^{j-1} \int_{C_{j}}|u(x)|^{2} d x\right)^{1 / 2},
$$

where $C_{j}=C\left(0,2^{j-2}, 2^{j-1}\right)$ if $j \geqslant 2$ and $C_{1}=B(0,1)$, and

$$
\|u\|_{B^{*}}=\sup _{R>1}\left(\frac{1}{R} \int_{\{|x|<R\}}|u(x)|^{2} d x\right)^{1 / 2} .
$$


The subspace $B^{* 0}=\left\{u \in B^{*}, \lim _{R \rightarrow+\infty} \frac{1}{R} \int_{|x|<R}|u(x)|^{2} d x=0\right\}$ is closed in $B^{*}$. In a similar manner, for $n \in \mathbb{N}$, we denote $B_{n}^{*}$ the set of functions $u \in H_{\text {loc }}^{n}$ such that all the successive derivatives, $\partial^{\alpha} u$, of $u$ for $|\alpha| \leqslant n$, belong to $B^{*}$. Its norm is naturally defined.

\section{Mathematical framework and the direct problem}

\subsection{Some well known results}

\subsubsection{The scattering amplitude}

Let $k>0$.

Definition 1. Let $u \in H_{\mathrm{loc}}^{1}$. The function $u$ is said to satisfy the Sommerfeld $k$-outgoing condition if $\left(\frac{\partial u}{\partial n}-i k u\right) \in$ $B^{* 0}$, where $n=x /|x|$. Such a function $u$ will be called a $k$-outgoing function.

A solution, say $u$, to Helmholtz equation

$$
-\Delta u(x)-k^{2} u(x)=0, \quad \text { for }|x| \geqslant R
$$

for some $R>0$, that satisfies the Sommerfeld $k$-outgoing condition admits an asymptotic expansion of the form

$$
u(x)=\frac{e^{i k|x|}}{|x|}\left(u_{\infty}\left(\frac{x}{|x|}\right)+\mathrm{O}\left(\frac{1}{|x|}\right)\right) \quad \text { as }|x| \mapsto \infty .
$$

The function $u_{\infty}$ is analytic on $S^{2}$ and is the so-called scattering amplitude of $u$. Note that $u_{\infty}=0$ implies that $u(x)=0$ for $|x| \geqslant R$ (see the Rellich Lemma, [1], p. 32).

\subsubsection{Limiting absorption principle}

We remind the reader of a well known limiting absorption principle that will be useful to us.

Proposition 1. Let us consider the operator $-\Delta: H^{2}\left(\mathbb{R}^{3}\right) \rightarrow L^{2}\left(\mathbb{R}^{3}\right)$ and, for $z \in \mathbb{C} \backslash[0,+\infty[$, its resolvent map $R(z): L^{2}\left(\mathbb{R}^{3}\right) \ni u \mapsto(-\Delta-z)^{-1} u$. Then

(i) The resolvent map can be extended for $z \in] 0,+\infty\left[\right.$ to a bounded operator $R^{+}(z): B \rightarrow B^{*}$ such that

$$
\forall k>0, \forall f, g \in B, \quad \lim _{z \rightarrow k^{2}, \operatorname{Im} z>0}(R(z) f, g)_{B^{*}, B}=\left(R^{+}\left(k^{2}\right) f, g\right)_{B^{*}, B} .
$$

(ii) Moreover, for $z \in \mathbb{C} \backslash\{0\}$ with $\operatorname{Im} z \geqslant 0, R^{+}(z)$ is a continuous operator from $B$ into $B_{2}^{*}$.

(iii) If $f \in B$ and $k>0$, the function $u=R^{+}\left(k^{2}\right) f$ is the unique $k$-outgoing solution of

$$
-\Delta u-k^{2} u=f \text {. }
$$

(iv) Let be $f \in B$ and $k, R>0$. If $\operatorname{supp} f \in B(0, R)$, the function $R^{+}\left(k^{2}\right)(f)$, that can be viewed as a $k$ outgoing solution to the homogeneous Helmholtz equation outside $B(0, R)$, admits the scattering amplitude given by the following expansion

$$
R^{+}\left(k^{2}\right) f(x)=\frac{e^{i k|x|}}{4 \pi|x|} \mathcal{F}(f)\left(k \frac{x}{|x|}\right)+\mathrm{O}\left(\frac{1}{|x|^{2}}\right)
$$

For a proof, see [2] (Theorem 14.3.2, pp. 238) for (i), (ii) and (iii). Points (iii) and (iv) are direct consequences of the following well known relation

$$
R^{+}\left(k^{2}\right) f(x)=\frac{1}{4 \pi} \int_{\mathbb{R}^{3}} \frac{e^{i k|x-y|}}{|x-y|} f(y) d y .
$$


Point (iii) can also be deduced from Theorem 14.3.4 in [2].

Point (iv) is also a consequence of the following

$$
\begin{aligned}
R^{+}\left(k^{2}\right) f(x) & =\int \frac{\mathcal{F}(f)(\xi)}{\left(|\xi|^{2}-k^{2}\right)} e^{i \xi \cdot x} d \xi \\
& =\int \frac{\mathcal{F}(f)(\xi)-\mathcal{F}(f)(k x /|x|)}{\left(|\xi|^{2}-k^{2}\right)} e^{i \xi \cdot x} d \xi+\mathcal{F}(f)\left(\frac{k x}{|x|}\right) \int \frac{1}{|\xi|^{2}-k^{2}} e^{i \xi \cdot x} d \xi .
\end{aligned}
$$

The first integral is small for large values of $|x|$ by standard stationary phase argument and the second is exactly the fundamental solution $e^{i k|x|} /(4 \pi|x|)$ of the Helmholtz operator. We deduce point (iv), and the advantage of this argument is that it can be generalized to more general operators.

\subsection{The direct problem}

We are interested here in solving (6) and (7). First, note that for $u, w \in H_{\mathrm{loc}}^{2}\left(\mathbb{R}^{3}\right)$, the two sides of (6) and (7) are well defined, as square-integrable functions.

We aim at obtaining functions $u, w \in H_{\mathrm{loc}}^{2}$ that solve (6) and (7), and also satisfy the Sommerfeld $k$-outgoing condition. Note that, from Proposition 1(iii), Eqs. (6) and (7) are strictly equivalent to

$$
u=R^{+}\left(k^{2}\right)\left[\theta F\left(u+\lambda e^{i k x \cdot d}\right)\right]
$$

and

$$
w=R^{+}\left(k^{2}\right)\left[\left(\left(w+\lambda e^{i k x \cdot d}\right) \varphi+i \psi \cdot \nabla\left(w+\lambda e^{i k x \cdot d}\right)\right)\left|\nabla\left(w+\lambda e^{i k x \cdot d}\right)\right|^{r}\left|w+\lambda e^{i k x \cdot d}\right|^{s}\right] .
$$

Thus, the solutions $u$ and $w$ must belong to $B_{2}^{*}$. The following result will enable us to uniquely define $u$ and $w$.

Proposition 2. Let $M>0$, fixed, and assume a priori $\|\theta\|_{\infty} \leqslant M$ (respectively $\|\varphi\|_{\infty},\|\psi\|_{\infty} \leqslant M$ ). Then

(i) $\exists \alpha>0$ such that (6) (respectively (7)) possesses a unique solution $u \in H_{\mathrm{loc}}^{2}$ (respectively $w \in H_{\mathrm{loc}}^{2}$ ) that satisfies $\|u\|_{B_{2}^{*}} \leqslant \alpha$ (respectively $\|w\|_{B_{2}^{*}} \leqslant \alpha$ ), provided that $\lambda \leqslant \alpha$.

(ii) Moreover, with the above conditions and notations, we have the following estimates

$$
\begin{aligned}
& \|u\|_{B_{2}^{*}} \leqslant C\|\theta\|_{\infty} \lambda^{p+1}, \\
& \|w\|_{B_{2}^{*}} \leqslant C\left(\|\varphi\|_{\infty}+\|\psi\|_{\infty}\right) \lambda^{r+s+1},
\end{aligned}
$$

where $C$ is a constant that does not depend on $\theta, \varphi, \psi, \lambda$ and $d \in S^{2}$.

Proof. We make use of the Banach contraction theorem. We set

$$
\begin{aligned}
& \Phi: B_{2}^{*} \rightarrow B_{2}^{*}, \\
& u \mapsto R^{+}\left(k^{2}\right)\left(\theta F\left(u+\lambda e^{i k x . d}\right)\right)
\end{aligned}
$$

and

$$
\begin{aligned}
& \Psi: B_{2}^{*} \rightarrow B_{2}^{*}, \\
& w \mapsto R^{+}\left(k^{2}\right)\left(\left(\varphi\left(w+\lambda e^{i k x \cdot d}\right)+i \psi \cdot \nabla\left(w+\lambda e^{i k x \cdot d}\right)\right)\left|\nabla\left(w+\lambda e^{i k x \cdot d}\right)\right|^{r}\left|w+\lambda e^{i k x \cdot d}\right|^{s}\right) .
\end{aligned}
$$

The proof involves the two following lemma and $C$ will always denote a positive constant that does not depend on $\theta, \varphi, \psi, \lambda$ and $d \in S^{2}$.

We have

Lemma 1 (stability). There is a constant $\alpha^{\prime}>0$ such that $\Phi\left[B_{B_{2}^{*}}\left(0, \alpha^{\prime}\right)\right] \subset B_{B_{2}^{*}}\left(0, \alpha^{\prime}\right)$ and $\Psi\left[B_{B_{2}^{*}}\left(0, \alpha^{\prime}\right)\right] \subset$ $B_{B_{2}^{*}}\left(0, \alpha^{\prime}\right)$ provided that $\lambda \leqslant \alpha^{\prime}$. 
Proof. We first consider Eq. (6). Let $\alpha^{\prime}>0$ (that will be fixed later) and assume $u \in B_{B_{2}^{*}}\left(0, \alpha^{\prime}\right)$. For technical reasons, we will assume that the real number $\alpha^{\prime}$ is less than 1. Then, via Proposition 1(ii)

$$
\|\Phi(u)\|_{B_{2}^{*}} \leqslant C\|\theta\|_{\infty}\left\|F\left(u+\lambda e^{i k x . d}\right)\right\|_{L^{2}(U)} .
$$

From the assumptions made on $F$, the boundedness of $U$, and the continuous imbedding

$$
H^{2}(U) \rightarrow C^{0}(\bar{U})
$$

$\left(C^{0}(\bar{U})\right.$ is the set of continuous functions on $\bar{U}$ ) there exists $C>0$ (that does not depend on $\lambda$ and $d$ since $\alpha^{\prime}<1$ ) such that

$$
\left\|F\left(u+\lambda e^{i k x \cdot d}\right)\right\|_{L^{2}(U)} \leqslant C\left(\|u\|_{B_{2}^{*}}^{p+1}+\lambda^{p+1}\right) .
$$

From (13) we thus obtain

$$
\|\Phi(u)\|_{B_{2}^{*}} \leqslant C_{0}\|\theta\|_{\infty}\left(\|u\|_{B_{2}^{*}}^{p+1}+\lambda^{p+1}\right) .
$$

We now check that the right-hand side member of (14) is smaller than $\alpha^{\prime}$ if $\alpha^{\prime}=\left(\frac{1}{2 C_{0} M}\right)^{1 / p}$.

For Eq. (7), the proof is similar (use the continuous imbedding $H^{1}(U) \subset L^{6}(U)$ for $\nabla u$ and note that $r \leqslant 2$ ). In particular it yields

$$
\|\Psi(w)\|_{B_{2}^{*}} \leqslant C_{0}\left(\|\varphi\|_{\infty}+\|\psi\|_{\infty}\right)\left(\|w\|_{B_{2}^{*}}^{r+s+1}+\lambda^{r+s+1}\right) .
$$

Lemma 2 (contraction). There is a constant $\alpha^{\prime \prime}>0$ such that $\Phi$ and $\Psi$ are $\frac{1}{2}$-contractions on $B_{B_{2}^{*}}\left(0, \alpha^{\prime \prime}\right)$ provided that $\lambda \leqslant \alpha^{\prime \prime}$.

Proof. Let $u, v \in B_{B_{2}^{*}}\left(0, \alpha^{\prime \prime}\right)$ and $\lambda \leqslant \alpha^{\prime \prime}$, where $\alpha^{\prime \prime}$ is supposed to be less than $\alpha^{\prime}$, and will be fixed later. We set $\tilde{u}(x)=u(x)+e^{i k x \cdot d}, \tilde{v}(x)=v(x)+e^{i k x \cdot d}$ and we have

$$
\|\Phi(u)-\Phi(v)\|_{B_{2}^{*}} \leqslant C\|\theta\|_{\infty}\|F(\tilde{u})-F(\tilde{v})\|_{L^{2}(U)},
$$

and

$$
\begin{aligned}
\|\Psi(u)-\Psi(v)\|_{B_{2}^{*}} \leqslant & C\|\varphi\|_{\infty}\left\|\tilde{u}|\tilde{u}|^{s}|\nabla \tilde{u}|^{r}-\tilde{v}|\tilde{v}|^{s}|\nabla \tilde{v}|^{r}\right\|_{L^{2}(U)} \\
& +C\|\psi\|_{\infty}\left\|\nabla \tilde{u}|\tilde{u}|^{s}|\nabla \tilde{u}|^{r}-\nabla \tilde{v}|\tilde{v}|^{s}|\nabla \tilde{v}|^{r}\right\|_{L^{2}(U)} .
\end{aligned}
$$

(a) Eq. (6). From the assumptions made on $F$ and $\varepsilon$, we can view $F$ as a differentiable map from $\mathbb{R}^{2} \sim \mathbb{C}$ into itself, and $\lim _{|z| \rightarrow 0} D F(z)=0$, where $D F$ is the $\mathbb{R}^{2}$-differential of $F$. Assume $\eta>0$. Provided that $\alpha^{\prime \prime}$ is small enough, we have from the hypothesis " $\left|\varepsilon^{\prime}(t)\right|=\mathrm{o}\left(1 / t^{p+1}\right)$ ",

$$
\sup _{|z| \leqslant(A+1) \alpha^{\prime \prime}}\|D F(z)\| \leqslant \eta
$$

where

- $\|D F(z)\|$ is the Euclidean norm of $D F(z)$, viewed as a linear operator from $\mathbb{R}^{2}$ into itself.

- $A$ is the norm of the continuous map

$$
\begin{aligned}
& B_{2}^{*} \rightarrow C^{0}(\bar{U}), \\
& \omega \mapsto \omega_{\mid U} .
\end{aligned}
$$


Hence, for $x \in \bar{U}$, we have $|\tilde{u}(x)|,|\tilde{v}(x)| \leqslant(A+1) \alpha^{\prime \prime}$, so

$$
|F(\tilde{u}(x))-F(\tilde{v}(x))| \leqslant \eta|u(x)-v(x)| ;
$$

hence

$$
\|F(\tilde{u}(x))-F(\tilde{v}(x))\|_{L^{2}(U)} \leqslant C \eta\|u-v\|_{B_{2}^{*}},
$$

and $C$ only depends on $R_{0}$.

From (16), we can deduce

$$
\|\Phi(u)-\Phi(v)\|_{B_{2}^{*}} \leqslant C M \eta\|u-v\|_{B_{2}^{*}(U)} .
$$

Choose $\eta=\frac{1}{2 C M}$, choose $\alpha^{\prime \prime}$ according to $\eta$, and the lemma is proven.

(b) Eq. (7). Here we have to use another method since $\nabla u$ is not necessarily a bounded function.

We have the following inequalities

$$
\begin{aligned}
& \forall x, y \in \mathbb{C}, \forall t \geqslant 1, \\
& \left.|| x\right|^{t}-|y|^{t}|\leqslant C| x-y\left|\left(|x|^{t-1}+|y|^{t-1}\right), \quad\right| x|x|^{t}-y|y|^{t}|\leqslant C| x-y \mid\left(|x|^{t}+|y|^{t}\right)
\end{aligned}
$$

and similar inequalities hold for $x, y \in \mathbb{C}^{3}$. We deduce, for $r, s \neq 0$ (if $r=0$ or $s=0$, the proof still holds with obvious modifications) the two following points.

(1) On the one hand,

$$
\begin{aligned}
\left\|\tilde{u}|\tilde{u}|^{s}|\nabla \tilde{u}|^{r}-\tilde{v}|\tilde{v}|^{s}|\nabla \tilde{v}|^{r}\right\|_{L^{2}(U)} \leqslant & C\|u-v\|_{\infty, U}\left(\|\tilde{u}\|_{\infty, U}^{s}+\|\tilde{v}\|_{\infty, U}^{s}\right)\|\nabla \tilde{u}\|_{L^{2 r}(U)}^{r} \\
& +C\|\tilde{v}\|_{\infty, U}^{s+1}\|\nabla u-\nabla v\|_{L^{6}(U)}\left(\left\||\nabla \tilde{u}|^{r-1}\right\|_{L^{3}(U)}+\left\||\nabla \tilde{v}|^{r-1}\right\|_{L^{3}(U)}\right) .
\end{aligned}
$$

Note that

$$
\left\||\nabla \tilde{u}|^{r-1}\right\|_{L^{3}(U)} \leqslant\|\nabla \tilde{u}\|_{L^{6}(U)}^{r-1}(\operatorname{Vol} U)^{\frac{3-r}{6}}
$$

and the above equality turns into

$$
\begin{aligned}
\left\|\tilde{u}|\tilde{u}|^{s}|\nabla \tilde{u}|^{r}-\tilde{v}|\tilde{v}|^{s}|\nabla \tilde{v}|^{r}\right\|_{L^{2}(U)} \leqslant & C\|u-v\|_{\infty, U}\left(\|\tilde{u}\|_{\infty, U}^{s}+\|\tilde{v}\|_{\infty, U}^{s}\right)\|\nabla \tilde{u}\|_{L^{2 r}(U)}^{r} \\
& +C\|\tilde{v}\|_{\infty, U}^{s+1}\|\nabla u-\nabla v\|_{L^{6}(U)}\left(\|\nabla \tilde{u}\|_{L^{6}(U)}^{r-1}+\|\nabla \tilde{v}\|_{L^{6}(U)}^{r-1}\right) .
\end{aligned}
$$

(2) On the other hand, we have

$$
\begin{aligned}
\left\|\nabla \tilde{u}|\tilde{u}|^{s}|\nabla \tilde{u}|^{r}-\nabla \tilde{v}|\tilde{v}|^{s}|\nabla \tilde{v}|^{r}\right\|_{L^{2}(U)} \leqslant & C\|\tilde{u}\|_{\infty, U}^{s}\|\nabla u-\nabla v\|_{L^{6}(U)}\left(\|\nabla \tilde{u}\|_{L^{3 r}(U)}^{r}+\|\nabla \tilde{v}\|_{L^{3 r}(U)}^{r}\right) \\
& +C\|\nabla \tilde{v}\|_{L^{6}(U)}^{r+1}\|u-v\|_{\infty, U}\left(\|\tilde{u}\|_{\infty, U}^{s-1}+\|\tilde{v}\|_{\infty, U}^{s-1}\right) .
\end{aligned}
$$

We conclude from (17) and the following Sobolev imbeddings (note that $r \leqslant 2$ )

$$
\begin{aligned}
& H^{1} \subset L^{t}, \quad t \leqslant 6, \\
& H^{2} \subset L^{\infty}
\end{aligned}
$$

that

$$
\|\Psi(u)-\Psi(v)\|_{B_{2}^{*}} \leqslant C\left(\|\varphi\|_{\infty}+\|\psi\|_{\infty}\right)\|u-v\|_{B_{2}^{*}}\left(\|\tilde{u}\|_{B_{2}^{*}}^{r+s}+\|\tilde{v}\|_{B_{2}^{*}}^{r+s}\right) \leqslant 4 C M\left(\alpha^{\prime \prime}\right)^{r+s}\|u-v\|_{B_{2}^{*}} .
$$

We conclude from (18) that $\Psi$ is a $\frac{1}{2}$-contraction as soon as $\alpha^{\prime \prime}$ is chosen small enough.

Going back to the proof of Proposition 2, we see that part (i) is a direct consequence of the two above lemmas, and the Banach contraction theorem. We only prove (11) (the proof is similar for (12), using (15)). 
We write (14) for $u \in B_{B_{2}^{*}}\left(0, \alpha^{\prime \prime}\right)$ and note that from the choice of $\alpha^{\prime}$ we have $\|u\|_{B_{2}^{*}}^{p+1} \leqslant \frac{1}{2 C_{0} M}\|u\|_{B_{2}^{*}}$. We conclude that $\|\Phi(u)\|_{B_{2}^{*}} \leqslant \frac{1}{2}\|u\|_{B_{2}^{*}}+C_{0} \lambda^{p+1}\|\theta\|_{\infty}$. Since the solution provided in (i) is a fixed point of $\Phi$, we obtain (11) which completes the proof.

\section{Behaviour of $u(x, d, \lambda)$ for $\lambda \rightarrow 0$}

Along the next sections, $\theta, \varphi$ and $\psi$ are supposed to satisfy an a priori estimate $\|\theta\|_{\infty},\|\varphi\|_{\infty},\|\psi\|_{\infty} \leqslant M$. Hence, thanks to Proposition $2, u(., d, \lambda)$ and $w(., d, \lambda)$ can be defined without ambiguity as solutions of (6) and (7) for small values of $\lambda$.

Here, we show that $u(x, d, \lambda)=\lambda^{p+1}\left(v(x, d)+\varepsilon_{0}(x, d, \lambda)\right)$ and $w(x, d, \lambda)=\lambda^{r+s+1}\left(w(x, d)+\varepsilon_{0}(x, d, \lambda)\right)$, where $\varepsilon_{0}(x, d, \lambda)$ converges to 0 uniformly for $d \in S^{2}$ and bounded $x$, if $\lambda \rightarrow 0$ and $v$ can easily be deduced from $\theta$, (respectively $\varphi$ and $\psi$ ).

Lemma 3. The following convergences hold in $B_{2}^{*}$ :

$$
\lim _{\lambda \rightarrow 0} \frac{u(., d, \lambda)}{\lambda^{p+1}}=R^{+}\left(k^{2}\right)\left(\theta e^{i k x . d}\right)
$$

and

$$
\lim _{\lambda \rightarrow 0} \frac{w(., d, \lambda)}{\lambda^{r+s+1}}=R^{+}\left(k^{2}\right)\left[k^{r}(\varphi-k d . \psi) e^{i k x \cdot d}\right] .
$$

Proof. (1) Eq. (6). From (9), we have

$$
\frac{u(x, d, \lambda, \theta)}{\lambda^{p+1}}=R^{+}\left(k^{2}\right)\left[\theta\left(\frac{u}{\lambda}+e^{i k x . d}\right)\left|\frac{u}{\lambda}+e^{i k x . d}\right|^{p}\left(1+\varepsilon\left(\left|u+\lambda e^{i k x . d}\right|\right)\right)\right]
$$

and we use (11) to prove that the term between square brackets [ ] in (19) converges to $\theta(x) e^{i k x . d}$ if $\lambda \rightarrow 0$, uniformly in $(x, d) \in U \times S^{2}$. Hence, by Proposition 1(ii), the lemma holds.

(2) Eq. (7). We divide both sides of (10) by $\lambda^{r+s+1}$, and the term $\frac{1}{\lambda^{r+s+1}}[]$ converges in $L^{2}(U)$ to $k^{r}(\varphi-k d . \psi) e^{i k x . d}$ (use (12)). Hence the proof follows as in the case of Eq. (6).

\section{The inverse problem}

This last section is devoted to the inverse problem announced in the Introduction. We state the uniqueness. We point out the case for which $\theta, \varphi$ and $\psi$ are spherical functions, and show that the hypothesis on the scattering data we need for Theorem 1 are optimal for the general case. For large $k$, we give an explicite way of reconstruction for $\theta$. Questions of stability are also raised.

\subsection{Main result}

First, note that for $\lambda$ sufficiently small, $u(x, d, \lambda, \theta)$ and $w(x, d, \lambda,(\varphi, \psi))$, solutions to (6) and (7), exist and are $k$-outgoing solutions to the homogeneous Helmholtz equation outside $U$. Hence these functions admit a scattering amplitude, denoted $u_{\infty}(x, d, \lambda, \theta)$ and $w_{\infty}(x, d, \lambda,(\varphi, \psi))$ (see Section 1.1.1). 


\subsubsection{Zero-order perturbation}

Here we are concerned with the Eq. (6).

Theorem 1. Suppose $\theta_{1}, \theta_{2} \in L^{\infty}$, with $\operatorname{supp} \theta_{j} \subset U$ and $\left\|\theta_{j}\right\|_{\infty} \leqslant M$ for $j=1,2$. For small values of $\lambda$, we denote $u(x, d, \lambda, \theta)$ the solution of $(6)$ and $u_{\infty}(x, d, \lambda, \theta)$ its scattering amplitude.

(i) Let $Z \subset S^{2}$ a non-finite countable subset, such that

$$
\forall d \in Z, \forall \hat{x} \in S^{2}, \quad \lim _{\lambda \rightarrow 0} \frac{u_{\infty}\left(\hat{x}, d, \lambda, \theta_{1}\right)}{\lambda^{p+1}}=\lim _{\lambda \rightarrow 0} \frac{u_{\infty}\left(\hat{x}, d, \lambda, \theta_{2}\right)}{\lambda^{p+1}} .
$$

Then $\theta_{1}=\theta_{2}$.

(ii) Moreover, assume that $\theta_{1}, \theta_{2}$ are radial functions (i.e., $\theta_{j}(x)=\theta_{j}^{*}(|x|)$ for $\left.x \in \mathbb{R}^{3}, j=1,2\right)$, and let $d_{0} \in S^{2}$ and $\gamma:[0,1] \rightarrow S^{2}$ a continuous path such that the function $t \mapsto \gamma(t) . d_{0}$ does not remain constant. If the following relation holds

$$
\forall t \in[0,1], \quad \lim _{\lambda \rightarrow 0} \frac{u_{\infty}\left(\gamma(t), d_{0}, \lambda, \theta_{1}\right)}{\lambda^{p+1}}=\lim _{\lambda \rightarrow 0} \frac{u_{\infty}\left(\gamma(t), d_{0}, \lambda, \theta_{2}\right)}{\lambda^{p+1}},
$$

then $\theta_{1}=\theta_{2}$.

\section{Proof.}

(i) Let $d \in S^{2}$, and $\lambda \in(0,+\infty)$ small enough. For $\theta=\theta_{1}, \theta_{2}$, note that from (19) and Proposition 1(iv), $u_{\infty}(\hat{x}, d, \lambda, \theta) / \lambda^{p+1}$ is given by the Fourier transform of

$$
\theta\left(\frac{u(x, d, \lambda, \theta)}{\lambda}+e^{i k x . d}\right)\left|\frac{u(x, d, \lambda, \theta)}{\lambda}+e^{i k x . d}\right|^{p}\left(1+\varepsilon\left(\left|u(x, d, \lambda, \theta)+\lambda e^{i k x . d}\right|\right)\right)
$$

calculated at $k \hat{x}$. Moreover the following family of functions

$$
x \mapsto \theta(x)\left(\frac{u(x, d, \lambda, \theta)}{\lambda}+e^{i k x . d}\right)\left|\frac{u(x, d, \lambda, \theta)}{\lambda}+e^{i k x . d}\right|^{p}\left(1+\varepsilon\left(\left|u(x, d, \lambda, \theta)+\lambda e^{i k x . d}\right|\right)\right)
$$

converges in $L^{2}\left(\mathbb{R}^{3}\right)$, uniformly in $d \in S^{2}$ as $\lambda \rightarrow 0$ and, as its support remains included in $\bar{U}$, its Fourier transform and all its derivatives converge uniformly on any bounded subset of $\mathbb{R}^{3}$. Thus, by Proposition 1(iv) and Lemma 3 , we conclude that

$$
\lim _{\lambda \rightarrow 0} \frac{u_{\infty}(\hat{x}, d, \lambda, \theta)}{\lambda^{p+1}}=\frac{1}{4 \pi} \mathcal{F}\left(\theta e^{i k x . d}\right)(k \hat{x})=\frac{1}{4 \pi} \mathcal{F}(\theta)(k(\hat{x}-d)) .
$$

Going back to the proof of (i), we see that, with the above remarks, the Fourier transform of $\theta=\theta_{1}-\theta_{2}$ is an analytic function, $g$, on $\mathbb{R}^{3}$ that cancels on $\left\{k(\hat{x}-d) ; \hat{x} \in S^{2}, d \in Z\right\}=\bigcup_{d \in Z} S(-k d, k)$. We now prove that $g$ is zero.

Consider a sequence $\left(d_{n}\right) \subset Z$ such that $d_{n} \rightarrow d_{\infty}$ and $\forall n \in \mathbb{N}, d_{n} \neq d_{\infty}$. We can suppose that $\delta=$ $\lim _{n \rightarrow \infty} \frac{d_{n}-d_{\infty}}{\left|d_{n}-d_{\infty}\right|}$ exists in $S^{2}$.

Let us choose $\hat{x} \in S^{2}$ such that $\delta . \hat{x} \neq 0$ and define

$$
\begin{aligned}
& f: \mathbb{R} \rightarrow \mathbb{C}, \\
& t \mapsto g\left(k \hat{x}-k d_{\infty}+t \delta\right) .
\end{aligned}
$$

We have $f(t)=0$ for every $t \in \mathbb{R}$ that satisfies

$\exists n \in \mathbb{N}, \quad\left|t \delta+k \hat{x}+k\left(d_{n}-d_{\infty}\right)\right|=k$, since $k \hat{x}-k d_{\infty}+t \delta \in S\left(-k d_{n}, k\right)$. 
We would like to show that the subset $\left\{t \in \mathbb{R} \exists n \in \mathbb{N},\left|t \delta+k \hat{x}+k\left(d_{n}-d_{\infty}\right)\right|=k\right\}$ is non-finite. Hence, consider the following smooth application

$$
\begin{aligned}
& F: \mathbb{R} \times S^{2} \rightarrow \mathbb{R}, \\
& (t, e) \mapsto\left|t \delta+k \hat{x}+k\left(e-d_{\infty}\right)\right|^{2}-k^{2} .
\end{aligned}
$$

Since $F\left(0, d_{\infty}\right)=0$ and $\left.\frac{\partial F\left(t, d_{\infty}\right)}{\partial t}\right|_{t=0}=2 k \delta . \hat{x} \neq 0$, the Implicit Functions Theorem ensures that, for sufficiently large values of $n \in \mathbb{N}$, there is a sequence $\left(t_{n}\right) \rightarrow 0$ such that $F\left(t_{n}, d_{n}\right)=0$. A straightforward calculation shows that

$$
F\left(0, d_{n}\right)=k^{2}\left|d_{n}-d_{\infty}\right|(2 \hat{x} . \delta+\mathrm{o}(1)) \quad \text { as } n \rightarrow \infty .
$$

We deduce that $F\left(0, d_{n}\right) \neq 0$ for sufficiently large values of $n$, hence $t_{n} \neq 0$. Since we have, $\forall n \in \mathbb{N}, f\left(t_{n}\right)=0$, we conclude that $f$ cancels on a bounded non-finite subset of $\mathbb{R}$. $f$ is analytic, we deduce that $f$ is zero. Hence, $g$ vanishes on the non-empty open subset $k\left(S^{2} \backslash\{\delta\}^{\perp}-d_{\infty}\right)+\mathbb{R} \delta$, since $\hat{x}$ is arbitrary in the two open half-sphere $S^{2} \backslash\{\delta\}^{\perp}$. Then $g$ is identically zero, and $\theta=0$.

(ii) As in (i), we obtain that $g$, the Fourier transform of $\theta=\theta_{1}-\theta_{2}$, vanishes on $\left\{k\left(\gamma(t)-d_{0}\right), t \in[0,1]\right\}$. We denote $m_{\gamma}=\min _{t \in[0,1]}\left|d_{0}-\gamma(t)\right|$ and $M_{\gamma}=\max _{t \in[0,1]}\left|d_{0}-\gamma(t)\right|$ and we have $m_{\gamma}<M_{\gamma}$ from the hypothesis of the theorem. Since $g$ is a radial function, and $\gamma$ is a continuous path, it is easily seen that $g$ cancels on $C\left(0, k m_{\gamma}, k M_{\gamma}\right)$. The function $g$ is analytic on $\mathbb{R}^{3}$, hence $g$ is identically zero, thus $\theta_{1}=\theta_{2}$.

Remark. An alternative proof of Theorem 1(i) that uses a geometrical approach has been suggested to us. Here is a sketch of this proof.

Let us start from (20). Note that tangential derivatives of all orders of $g$ vanish on $S\left(-k d_{\infty}, k\right)$. All spheres $S\left(-k d_{n}, k\right)$ pass through 0 and are not tangential there to $S\left(-k d_{\infty}, k\right)$ and to each other, because the $d_{n}$ are not colinear. Choose a sequence $\left(e_{m}\right) \subset S\left(-k d_{\infty}, k\right), e_{m} \neq 0$, such that $\left(e_{m}\right) \rightarrow 0$. Let $L_{m}$ be the line $\left\{e_{m}+t d, t \in \mathbb{R}\right\}$. Then every $L_{m}$ intersects all spheres $S\left(-k d_{n}, k\right)$ for large $n$, and $g$ is 0 at the points of intersection. So, from Rolle Theorem, for every order $p \in \mathbb{N}$, the directional derivative of $g$ of order $p$ along $L_{m}$ vanishes at a point $Y_{m, p} \in L_{m}$. The direction of $L_{m}$ is $d_{\infty}$, that is transversal to $S\left(-k d_{\infty}, k\right)$ at 0 , and $Y_{m, p} \rightarrow 0$ when $m \rightarrow \infty$.

Hence, since $d_{\infty}$ is transversal to $S\left(-k d_{\infty}, k\right)$ at 0 , all derivatives of $g$ of all orders vanish at 0 . By analyticity, $g=0$.

\subsubsection{First-order perturbation}

We extend Theorem 1 to Eq. (7) with stronger hypothesis.

Theorem 2. Suppose $\varphi_{1}, \varphi_{2} \in L^{\infty}\left(\mathbb{R}^{3}, \mathbb{R}\right), \psi_{1}, \psi_{2} \in L^{\infty}\left(\mathbb{R}^{3}, \mathbb{R}^{3}\right)$ with $\operatorname{supp} \varphi_{j}$, $\operatorname{supp} \psi_{j} \subset U$ and $\left\|\varphi_{j}\right\|_{\infty}$, $\left\|\psi_{j}\right\|_{\infty} \leqslant M$ for $j=1,2$.

For small values of $\lambda$, we denote $w(x, d, \lambda, \theta)$ the solution of (7) and $u_{\infty}(x, d, \lambda, \theta)$ its scattering amplitude.

(i) Assume that

$$
\forall \hat{x}, d \in S^{2}, \quad \lim _{\lambda \rightarrow 0} \frac{w_{\infty}\left(\hat{x}, d, \lambda,\left(\varphi_{1}, \psi_{1}\right)\right)}{\lambda^{r+s+1}}=\lim _{\lambda \rightarrow 0} \frac{w_{\infty}\left(\hat{x}, d, \lambda,\left(\varphi_{2}, \psi_{2}\right)\right)}{\lambda^{r+s+1}},
$$

then $\left(\varphi_{1}, \operatorname{div} \psi_{1}\right)=\left(\varphi_{2}, \operatorname{div} \psi_{2}\right)$, in the sense of distributions.

(ii) Moreover, assume that $\left(\varphi_{j}, \psi_{j}\right)$ are radial functions (i.e., $\varphi_{j}(x)=\varphi_{j}^{*}(|x|)$ and $\psi_{j}(x)=\psi_{j}^{*}(|x|) \frac{x}{|x|}, x \neq 0$ ) and let $d_{0} \in S^{2}$ and $\gamma:[0,1] \rightarrow S^{2}$ a continuous path such that the function $t \mapsto \gamma(t) . d_{0}$ does not remain constant. If the following relation holds

$$
\forall t \in[0,1], \quad \lim _{\lambda \rightarrow 0} \frac{w_{\infty}\left(\gamma(t), d_{0}, \lambda,\left(\varphi_{1}, \psi_{1}\right)\right)}{\lambda^{r+s+1}}=\lim _{\lambda \rightarrow 0} \frac{w_{\infty}\left(\gamma(t), d_{0}, \lambda,\left(\varphi_{2}, \psi_{2}\right)\right)}{\lambda^{r+s+1}},
$$

then $\left(\varphi_{1}, \psi_{1}\right)=\left(\varphi_{2}, \psi_{2}\right)$. 
Remark. Concerning (i), the proof will show that $\operatorname{div} \psi_{1}=\operatorname{div} \psi_{2}$ is the most one can obtain, unless $\psi_{1}$ and $\psi_{2}$ are supposed to be potential vector fields; more precisely, suppose that $\Pi_{1}, \Pi_{2}$ are distributions such that $\psi_{j}=\nabla \Pi_{j}$ on $\mathbb{R}^{3}$ for $j=1,2$. Then, we can conclude in (i) that $\psi_{1}=\psi_{2}$. Note that under the assumptions in point (ii) of the theorem, $\psi_{1}$ and $\psi_{2}$ are potential vector fields, therefore it is not surprising that one obtains uniqueness for $\psi$, and not only for $\operatorname{div} \psi$.

\section{Proof of Theorem 2.}

(i) Like for (20), we have

$$
\forall \hat{x}, d \in S^{2}, \quad \lim _{\lambda \rightarrow 0} \frac{w_{\infty}(\hat{x}, d, \lambda,(\varphi, \psi))}{k^{r} \lambda^{r+s+1}}=\frac{1}{4 \pi}(\mathcal{F}(\varphi)(k(\hat{x}-d))-k \mathcal{F}(\psi)(k(\hat{x}-d)) . d) .
$$

Now, we would like to set $y=k(\hat{x}-d)$ in the above equation. Consequently, we define, for $y \in \mathbb{R}^{3}$ such that $0<|y|<2 k$, the subset $\mathcal{C}_{y}=\left\{\left.d \in S^{2}\left|d . y=-\frac{1}{2 k}\right| y\right|^{2}\right\}$.

Note that $\mathcal{C}_{y}$ is the 1 -dimensional sphere contained in a plane orthogonal to $y$, centered at $-\frac{y}{2 k}$ and of radius $\sqrt{1-|y|^{2} / 4 k^{2}}$.

From (21) applied for $\varphi=\varphi_{1}-\varphi_{2}$ and $\psi=\psi_{1}-\psi_{2}$, we obtain

$$
\forall d \in \mathcal{C}_{y}, \quad \mathcal{F}(\varphi)(y)-k \mathcal{F}(\psi)(y) . d=0
$$

(note that $d \in \mathcal{C}_{y}$ implies that $y=k(\hat{x}-d)$ for a convenient choice of $\hat{x} \in S^{2}$ ).

For $y \in B(0,2 k) \backslash\{0\}$, we deduce from (22) that the angle between $\mathcal{F}(\psi)(y)$ and $d \in \mathcal{C}_{y}$ is constant and consequently, $\mathcal{F}(\psi)(y)=\frac{y \cdot \mathcal{F}(\psi)(y)}{|y|} \frac{y}{|y|}$ for $y \in B(0,2 k) \backslash\{0\}$. This last equality remains valid for $y \in \mathbb{R}^{3} \backslash\{0\}$ (analyticity).

From (22), the definition of $\mathcal{C}_{y}$ and the colinearity of $\mathcal{F}(\psi)(y)$ and $y$ we deduce

$$
\forall y \in B(0,2 k) \backslash\{0\}, \quad \mathcal{F}(\varphi)(y)+\frac{1}{2} y \cdot \mathcal{F}(\psi)(y)=0 .
$$

Thanks to an argument of analyticity, we actually obtain

$$
\forall y \in \mathbb{R}^{3}, \quad \mathcal{F}(\varphi)(y)+\frac{1}{2} y \cdot \mathcal{F}(\psi)(y)=0 .
$$

Note that the left-hand side of (23) is the Fourier transform of $\varphi-\frac{i}{2} \operatorname{div} \psi$. We conclude by extracting the real and imaginary parts of this last equation.

(ii) We have

$$
\forall t \in[0,1], \quad \mathcal{F}(\varphi)\left(k\left(\gamma(t)-d_{0}\right)\right)-k \mathcal{F}(\psi)\left(k\left(\gamma(t)-d_{0}\right)\right) \cdot d_{0}=0 .
$$

A straightforward calculation shows that $\forall \xi \in \mathbb{R}^{3} \backslash\{0\}, \mathcal{F}(\psi)(\xi)=\tau(\xi) \frac{\xi}{|\xi|}$ where $\tau(\xi)$ is a radial function

$$
\tau(\xi)=\frac{4 i \pi}{|\xi|^{2}} \int_{0}^{+\infty}\left(\psi_{1}^{*}(t)-\psi_{2}^{*}(t)\right)[t|\xi| \cos (t|\xi|)-\sin (t|\xi|)] d t .
$$

From (24), we have

$$
\forall t \in[0,1], \quad \mathcal{F}(\varphi)\left(k\left(\gamma(t)-d_{0}\right)\right)-k \tau\left(k\left|\gamma(t)-d_{0}\right|\right) \frac{\left(\gamma(t)-d_{0}\right) \cdot d_{0}}{\left|\gamma(t)-d_{0}\right|}=0 .
$$

Since $\gamma(t) \cdot d_{0}=1-\frac{1}{2}\left|\gamma(t)-d_{0}\right|^{2}$ we finally obtain

$$
\forall t \in[0,1], \quad \mathcal{F}(\varphi)\left(k\left(\gamma(t)-d_{0}\right)\right)+\frac{k}{2} \tau\left(k\left|\gamma(t)-d_{0}\right|\right)\left|\gamma(t)-d_{0}\right|=0 .
$$


Now, use the same arguments as those used for Theorem 1(ii), with $g$ replaced by the radial function

$$
x \mapsto \mathcal{F}(\varphi)(x)+\frac{|x|}{2} \tau(|x|),
$$

to conclude that

$$
\forall y \in \mathbb{R}^{3}, \quad \mathcal{F}(\varphi)(y)+\frac{|y|}{2} \tau(|y|)=0 .
$$

Note that the left-hand side of the above equality is the Fourier transform of $\varphi-\frac{i}{2} \operatorname{div} \psi$. One concludes, by extracting the real and imaginary part of this last equation, that $\varphi=0$ and $\operatorname{div} \psi=0$.

It yields that $\psi_{1}^{*}(x)=\psi_{2}^{*}(x)+\frac{C}{|x|^{2}}$ for some constant $C$ and $x \neq 0$. The functions $\psi_{1}^{*}$ and $\psi_{2}^{*}$ are bounded, which implies that $C=0$, hence $\psi_{1}^{*}=\psi_{2}^{*}$.

\subsection{Zero-order perturbation: some remarks}

\subsubsection{Scattering data}

Concerning the point (i) of Theorem 1 the hypothesis " $Z$ is a countable set" is optimal. More exactly, if $d_{1}, \ldots, d_{N} \in S^{2}$, there exists a function $\theta \neq 0$ such that $\lim _{\lambda \rightarrow 0} u_{\infty}\left(\hat{x}, d_{j}, \lambda, \theta\right) / \lambda^{p+1}=0$ for $j=1, \ldots, N$. In order to construct such a function $\theta$, consider $u \in C_{c}^{\infty}(U)$ such that $\left[\prod_{n=1}^{N}\left(\Delta+2 i k d_{n} . \nabla\right)\right] u \neq 0$ on $U$ (choose an ad hoc polynomial function and use a cut-off function) and set $\theta=\left[\prod_{n=1}^{N}\left(\Delta+2 i k d_{n} . \nabla\right)\right] u$; its Fourier transform vanishes on $\bigcup_{n=1}^{N} S\left(-k d_{n}, k\right)$. It follows that

$$
\lim _{\lambda \rightarrow 0} \frac{u_{\infty}\left(\hat{x}, d_{j}, \lambda, \theta\right)}{\lambda^{p+1}}=\lim _{\lambda \rightarrow 0} \frac{u_{\infty}\left(\hat{x}, d_{j}, \lambda, 0\right)}{\lambda^{p+1}}
$$

whereas $\theta \neq 0$.

\subsubsection{Stability}

In this section, we show that the inverse problem for which we have proven uniqueness in Theorem 1 is not stable with respect to the scattering data, for any order of regularity.

In fact, the knowledge of

$$
(\hat{x}, d) \mapsto \lim _{\lambda \rightarrow 0} \frac{u_{\infty}\left(\hat{x}, d, \lambda, \theta_{1}\right)}{\lambda^{p+1}}-\lim _{\lambda \rightarrow 0} \frac{u_{\infty}\left(\hat{x}, d, \lambda, \theta_{2}\right)}{\lambda^{p+1}}
$$

for $\hat{x}, d \in S^{2}$ is equivalent to the knowledge of the Fourier transform of $\theta$ on $B(0,2 k)$. This will be detailed in the Section 3.2.3. For each $n, p \in \mathbb{N}$, one cannot however find any constant $C_{n, p}$ such that

$$
\forall \theta \in C_{c}^{\infty}(U), \quad\|\theta\|_{H^{-p}} \leqslant C_{n, p}\|\mathcal{F}(\theta)\|_{H^{n}(B(0,2 k))} .
$$

If such a constant exists, then consider any distribution $\Phi \in H^{-p-1}$ such that $\Phi \notin H^{-p}$, with $\operatorname{supp} \Phi \subset U$. Choose a sequence $\left(\Phi_{l}\right)_{l} \subset C_{c}^{\infty}(\widetilde{U})$ that converges in $H^{-p-1}$ to $\Phi$, where $\widetilde{U}$ is a bounded open set containing $U$. We deduce that the sequence $\left(\mathcal{F}\left(\Phi_{l}\right)\right)_{l}$ converges in $H^{n}(B(0,2 k))$ since $\widetilde{U}$ is bounded (note that $H^{r, s}\left(\mathbb{R}^{3}\right)$ and $H^{s, r}\left(\mathbb{R}^{3}\right)$ are isomorphic by the Fourier transform), hence from (25), we could conclude that $\left(\Phi_{l}\right)_{l}$ converges in $H^{-p}$, that is $\Phi \in H^{-p}$, which is a contradiction with the choice of $\Phi$.

\subsubsection{Approximate reconstruction for $\theta$}

Here, we assume that $\lim _{\lambda \rightarrow 0} u_{\infty}(\hat{x}, d, \lambda, \theta) / \lambda^{p+1}$ is known for all values of $(\hat{x}, d) \in S^{2} \times S^{2}$. Theorem 1 only yields uniqueness for $\theta$, but does not provide a reconstruction. Note that to reconstruct $\theta$, one needs to 
know its Fourier transform on $B(0,2 k)$. However, Section 3.2.2 shows that there is no hope for stability in this reconstruction. More precisely, we have

$$
\lim _{\lambda \rightarrow 0} \frac{u_{\infty}(\hat{x}, d, \lambda, \theta)}{\lambda^{p+1}}=\mathcal{F}(\theta)(k(\hat{x}-d))
$$

and the application $(\hat{x}, d) \mapsto k(\hat{x}-d)$ maps $S^{2} \times S^{2}$ onto $B(0,2 k)$. Thus, for large $k$ the knowledge of the scattering data $\lim _{\lambda \rightarrow 0} u_{\infty}(\hat{x}, d, \lambda, \theta) / \lambda^{p+1}$ is nearly equivalent to the knowledge of $\theta$ via the inverse Fourier transform, which will be shown by the next proposition.

In practice, one has to agree upon a quantity $\varepsilon>0$ that measures and control the lack of precision of the measurements; one can consider $x \sim y$ as soon as $|x-y| \leqslant \varepsilon$. The following proposition shows that, for large $k$ and small $\varepsilon, \theta$ can be reconstructed approximately from the scattering data.

Proposition 3. Let $\theta \in L^{\infty}\left(\mathbb{R}^{3}\right)$ with $\operatorname{supp} \theta \subset B\left(0, R_{0}\right) \subset \mathbb{R}^{3}$ and $\|\theta\|_{\infty} \leqslant M$. We define the function $w: B(0,2 k) \rightarrow \mathbb{C}$ by $w(y)=\lim _{\lambda \rightarrow 0} u_{\infty}(\hat{x}, d, \lambda, \theta) / \lambda^{p+1}$, and $w$ is defined without ambiguity if $y=k(\hat{x}-$ $d), \hat{x}, d \in S^{2}$. Let $\left.\varepsilon>0, q \in\right] 1, \frac{3}{2}\left[\right.$ and suppose the a priori estimate $\|\theta\|_{W^{1,1}} \leqslant M^{\prime}$ holds. Let be $v_{\varepsilon} \in$ $L^{\infty}(B(0,2 k))$ such that $\left|v_{\varepsilon}(y)-w(y, \theta)\right| \leqslant \varepsilon \forall y \in B(0,2 k)$.

Then, there exists $C>0$ that only depends on $M, M^{\prime}, U$ and $q$ (and does not depend on $\theta, k, \varepsilon$ ), such that the following estimate holds

$$
\left\|\theta(y)-\left(\frac{1}{2 \pi}\right)^{3} \int_{|x|<2 k} e^{i k x \cdot y} v_{\varepsilon}(x) d x\right\|_{L_{y}^{q}(U)} \leqslant C\left(\frac{1}{k^{\frac{3}{q}-2}}+\varepsilon k^{3-\frac{3}{q}}\right) .
$$

Proof (sketch). Note that there exists $C$ that only depends on $M^{\prime}$ such that $|\mathcal{F}(\theta)(x)| \leqslant \frac{C}{1+|x|}$. Thus, for $y \in B(0,2 k)$, we have $\left|v_{\varepsilon}(y)-\mathcal{F}(\theta)(y)\right| \leqslant \varepsilon$ and for $|x|>2 k$, we have $\left|v_{\varepsilon}(y)-\mathcal{F}(\theta)(y)\right| \leqslant \frac{C}{1+|x|}$ if $v_{\varepsilon}$ is extended by 0 outside $B(0,2 k)$.

Now, use the boundedness of the Inverse Fourier transform extended from $L^{\frac{q}{q-1}}$ to $L^{q}$ to complete the proof with straightforward calculations.

Note that the real number $k$ has been introduced to obtain the time-harmonic solutions to (3); we can choose arbitrary large values of $k$ that yields high-frequency solutions. In practice, it would be convenient to consider $k \sim \frac{1}{\varepsilon}$, since $k=k_{\min }=\frac{3-2 q}{3} \frac{1}{\varepsilon}$ minimizes the right-hand side of (26). Then Proposition 3 implies that

$$
\left\|\theta(y)-\left(\frac{1}{2 \pi}\right)^{3} \int_{|x|<2 k} e^{i k x \cdot y} v_{\varepsilon}(x) d x\right\|_{L_{y}^{q}(U)} \leqslant C \varepsilon^{\frac{3}{q}-2}
$$

for $k \sim 1 / \varepsilon$.

In practice, one has to use an incident wave with a wavelenght of the same order as $\varepsilon$, that measures out the uncertainty of the measurement.

Note that for $\varepsilon=0$ (i.e, $v_{\varepsilon}$ is exactly the scattering datum, that is there is no uncertainty), formula (26) shows that $\theta$ can be exactly reconstructed by letting $k \rightarrow+\infty$.

\subsubsection{Generalisation}

In this paragraph, a nonlinear Helmholtz equation with a more general form than (1) is studied. We consider a sequence $\left(a_{n}\right)_{n} \subset L^{\infty}\left(\mathbb{R}^{3}\right)$, with supp $a_{n} \subset U$, such that the series $\sum_{n=1}^{+\infty}\left\|a_{n}\right\|_{L^{\infty}} \rho^{n}$ converges for some $\rho>0$. Then, we define the following function:

$$
S:(x, z) \mapsto \sum_{n=1}^{+\infty} a_{n}(x)|z|^{n}
$$


that is defined for $x \in \mathbb{R}^{3}$ and for each complex number $z$ such that $|z| \leqslant \rho$. We are interested in generalizing the results proven in the last two sections for the equation

$$
-\Delta v-k^{2} v=v S(x,|v|)
$$

with $k>0$.

For $d \in S^{2}$ and small values of $\lambda \geqslant 0$, we search for solutions $v$ to (28) of the form

$$
v(x, d, \lambda)=\lambda e^{i k x \cdot d}+u(x, d, \lambda), \quad \lambda \geqslant 0, d \in S^{2},
$$

where $u$ satisfies the outgoing Sommerfeld condition. The inverse problem we propose is the identification of the function $S$ from the knowledge of the behaviour of the scattering amplitude $u_{\infty}(\hat{x}, d, \lambda)$ as $\lambda \rightarrow 0$. We have to note that Eq. (1) is a particular case of (28) if $\varepsilon=0$ and $p \in \mathbb{N}$. Using methods similar to those used for Eq. (1), we obtain

Theorem 3. (i) There is a constant $\alpha>0$ such that Eq. (28) admits a unique solution $u \in B_{B_{2}^{*}}(0, \alpha)$ provided that $\lambda \leqslant \alpha$.

(ii) Moreover, there is a countable family $\left(u_{n}(., d)\right)_{n \geqslant 2} \subset B_{2}^{*}$ such that, for any $n \geqslant 2$, we have

$$
\lim _{\lambda \rightarrow 0^{+}} \frac{u(x, d, \lambda)-\sum_{j=2}^{n} u_{j}(x, d) \lambda^{j}}{\lambda^{n}}=0
$$

where the limit is taken in the space $B_{2}^{*}$.

(iii) Let us consider two functions $S_{1}$ and $S_{2}$ such that

$$
S_{1}:(x, z) \mapsto \sum_{n=1}^{+\infty} a_{n}(x)|z|^{n}
$$

and

$$
S_{2}:(x, z) \mapsto \sum_{n=1}^{+\infty} b_{n}(x)|z|^{n}
$$

and the solutions $u$ and $v$ of (28) with $S$ respectively replaced by $S_{1}$ and $S_{2}$, defined for small values of $\lambda$ via the part (i). We define the coefficients of the development in $\lambda$ of the solutions $u$ and $v$, that is, respectively, the families $\left\{u_{j}\right\}_{j} \geqslant 2$ and $\left\{v_{j}\right\}_{j} \geqslant 2$ that are $k$-outgoing solutions to the homogeneous Helmholtz equation outside $U$. Now, suppose that there is a non-finite countable subset $Z \subset S^{2}$ such that for each $\hat{x} \in S^{2}$ and $d \in Z$, the equalities of the scattering amplitudes $u_{j, \infty}(\hat{x}, d)=v_{j, \infty}(\hat{x}, d)$ hold for every integer $j \geqslant 2$. Then $S_{1}=S_{2}$.

This theorem can be proven using methods similar to those used in the last sections.

\section{Acknowledgement}

I would like to thank the referees for the improvments they have suggested for this work. I am also grateful to Y. Dermenjian and J. Le Rousseau for the interest they have shown for the manuscript.

\section{References}

[1] D. Colton, R. Kreiss, Inverse Acoustic and Electromagnetic Scattering Theory, Springer, 1998.

[2] L. Hörmander, The Analysis of Linear Partial Differential Operators II, Springer-Verlag, 1983. 
[3] V. Isakov, A. Nachmann, Global uniqueness for a 2-dimensional semilinear elliptic problem, Trans. Amer. Math. Soc. 347 (1995) $3375-$ 3390.

[4] V. Isakov, J. Sylvester, Global uniqueness for a semilinear elliptic inverse problem, CPAM 47 (1994) 1403-1410.

[5] A. Nachmann, Reconstruction from boundary measurement, Ann. Math. 128 (1988) 531-577.

[6] W.A. Strauss, Nonlinear scattering theory, in: J.A. La Vita, J.-P. Marchand (Eds.), Scattering Theory in Mathematical Physics, Reidel, Dordrecht, 1974.

[7] R. Weder, Inverse scattering for the nonlinear Schrödinger equation II. Reconstruction of the potential and the nonlinearity in the multidimensional case, Proc. Amer. Math. Soc. 129 (12) (2002) 3637-3645. 